\title{
RESEARCH HIGHLIGHTS 2007
}

\section{As an end of the year round-up, we asked Nature's editors to nominate favourite papers published elsewhere this year. For a pick of favourites from Nature itself, see page viii.}

\section{MOLECULAR BIOLOGY}

\section{Stem cell success}

Cell 131, 861-872 (2007); Nature Biotechnol. doi:10.1038/nbt1374 (2007); Science doi:10.1126/ science.1151526 (2007)

In 2006, Shinya Yamanaka and Kazutoshi Takahashi of Kyoto University in Japan successfully prepared stem-like cells, which can develop into almost any of the body's cell types, from adult mouse skin cells. The technique involved 'reprogramming' the skin cells by introducing four specific genes using retroviruses, and provided a means of obtaining stem-like cells without using eggs or destroying embryos.

In 2007, Yamanaka and his colleagues went one better, successfully reproducing the experiment with human skin cells. The recipe works because the additional genes function as high-level regulators, turning on other genes and thereby setting off a cascade of intracellular changes.

Later, the group reported that it had eliminated the cancer-causing gene c-Myc from the list of introduced genes, a research boon along the path towards patientmatched stem-cell therapies. James Thomson of the University of Wisconsin-Madison and his co-workers also published a c- $M y c$-free method.

\section{CANCER}

\section{Aid to resistance}

Science doi:10.1126/science.1141478 (2007)

The drug gefitinib is at first an effective treatment for certain types of lung cancer, but most cancers become resistant to it. New research provides insight into one mechanism by which this happens.

Gefitinib inhibits an enzyme known as EGFR kinase, and around half of the resistance cases are due to mutations in this target. Among the rest, Pasi Jänne of the Dana-

Farber Cancer Institute in Boston, Massachusetts, and his colleagues found that some resistant cells showed increased expression of a tyrosine-kinase receptor named MET. This activates

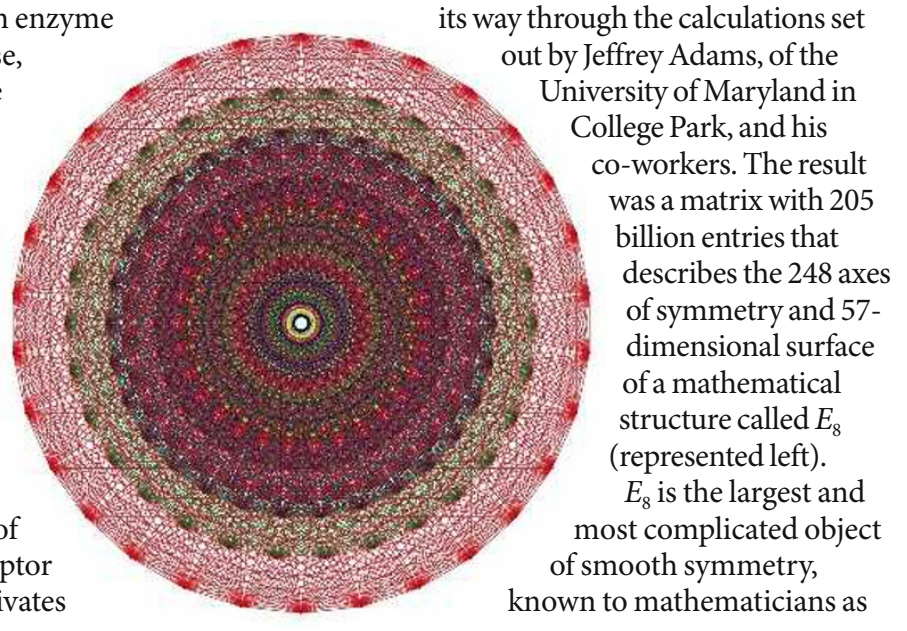
its way through the calculations set t by Jeffrey Adams, of the niversity of Maryland in College Park, and his co-workers. The result was a matrix with 205 billion entries that describes the 248 axes of symmetry and 57dimensional surface of a mathematical structure called $E_{8}$ (represented left). $E_{8}$ is the largest and most complicated objec
of smooth symmetry, known to mathematicians as

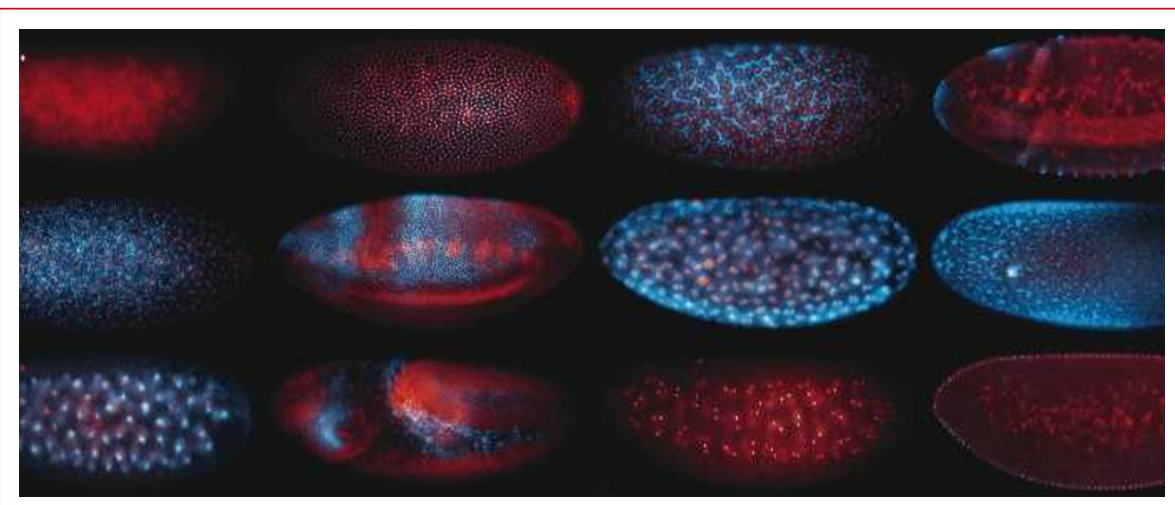

\section{RNA on the move}

Cell 131, 174-187 (2007)

Moving bulky proteins around a cell takes a lot of energy. These costs can be cut by moving a single messenger RNA (mRNA) and then producing many copies of its encoded protein where they are needed. Henry Krause of the University of Toronto and his colleagues have revealed that this happens to a much greater extent than expected in the early fruitfly (Drosophila melanogaster) embryo. The researchers followed the movements of mRNAs during the first 4.5 hours of embryonic development. They found that $71 \%$ of the mRNAs in their sample were moved to specific places.

The monitored mRNAs were targeted to about 35 different regions, and each type was always sent to the same places. Furthermore, the distribution of proteins closely matched patterns of mRNA localization. These results suggest that mRNA localization could regulate many cellular functions. the same signalling pathway that EGFR kinase triggers - a pathway that mediates cell survival.

The researchers found that the MET gene had multiplied rather than mutated, and that inhibiting MET restored the cells' sensitivity to gefitinib.

\section{MATHEMATICS}

\section{The 248th dimension}

www.liegroups.org

It took 77 hours for a supercomputer to crunch an 'exceptional Lie group'. The team's main challenge was to work out how to condense the sums required so as to make them small enough for a supercomputer to handle, a feat achieved by invoking the 'Chinese remainder theorem.

\section{MAGNETIC IMAGING}

\section{Getting to the point}

Nature Nanotechnol. 2,301-306 (2007)

The resolution achieved by magnetic resonance imaging (MRI) has improved 60,000 -fold, thanks to developments that couple MRI with atomic force microscopy.

In magnetic atomic force microscopy, a sample, in the form of a thin film, is placed on an ultrasensitive cantilever that is suspended above a conical magnetic tip. The tip's strong magnetic field means that changes in the spin of atomic nuclei in the sample change the way the cantilever oscillates.

John Mamin, at IBM's Almaden Research Center in San Jose, California, and his colleagues have developed highly magnetized tips with extremely sharp points, which generate large magnetic-field gradients. This allows them to achieve a resolution of 90 nanometres, making the system vastly more sensitive. 


\section{PLANETARY SCIENCE The whole of the Moon}

Earth Planet. Sci. Lett.

262, 438-449 (2007)

The consensus that

Earth's satellite was

created when a planet sometimes called Theia hit the early Earth has been tormented by a paradox for the past 30 years. Although computer simulations of the impact suggest that most of the Moon's mass came from Theia, its oxygen-isotope composition is very similar to that of Earth, suggesting that in fact it broke off from our home planet.

Kaveh Pahlevan and David Stevenson of the California Institute of Technology in Pasadena believe that their model resolves this contradiction. They propose that the massive impact some 4.5 billion years ago vaporized most of both Earth and Theia. The disk of magma that eventually became the Moon remained linked to Earth by a wash of silicate vapour. Within this vapour, turbulent mixing evened out the isotopic differences between Earth and Theia before the Moon condensed out, the researchers suggest.

\section{QUANTUM CRYPTOGRAPHY}

\section{Canary communication}

Nature Phys. doi:10.1038/nphys629 (2007) Physicists demonstrated the ability to distribute a quantum 'key' over a record 144 kilometres, the distance between two telescopes in the Canary Islands.

Anton Zeilinger and Rupert Ursin of the University of Vienna in Austria and their colleagues used a laser to produce two photons 'entangled' in such a way that a measurement of one influences measurements of the other. Quantum cryptography makes use of this effect to distribute cryptographic keys no eavesdropper could intercept without being detected.

While one photon was measured on the island of La Palma, the second was picked up by a telescope on the island of Tenerife. The team hopes that this advance will one day lead to satellite-based quantum communication.

\section{PARTICLE PHYSICS}

\section{Hard-core calculations}

Phys. Rev. Lett. 99, 022001 (2007)

Why do protons and neutrons stick close enough to form an atomic nucleus but never merge into a formless soup of their constituent quarks?

In July, a group of

Japanese physicists

answered this question by publishing firstprinciples calculations of the particles' internal dynamics.

The force between nuclear particles has long been known to be attractive at moderate distances but repulsive at

very close quarters, yet quantitative explanations of this in terms of the behaviour of the particles' constituent quarks have proved elusive.

Now Noriyoshi Ishii of the Universities of Tsukuba and Tokyo and his colleagues have used sophisticated algorithms and massively powerful computers to derive the familiar form of the nuclear force from the quantum field theory of quark interactions, called quantum chromodynamics. The achievement is both a computational tour de force and a triumph for theory.

\section{ECOLOGY}

\section{Hot threesome}

Science 315, 513-515 (2007)

A virus within a fungus is vital if that fungus and the grass it lives in are to survive scalding soil temperatures, according to work by Marilyn Roossinck of the Samuel Roberts Noble Foundation in Ardmore, Oklahoma, and her colleagues. They studied Dichanthelium lanuginosum, which grows in soils heated by geothermal activity in America's Yellowstone National Park (pictured below). Plants that lack the fungus Curvularia protuberata cannot survive in soils above $38^{\circ} \mathrm{C}$.

The researchers detected a virus inside the fungus. When plants were infected by a fungus that had been completely 'cured' of the virus, they shrivelled and died at high temperatures. Restoring the virus restored the system's heat tolerance. The virus-fungus combination also protected some tomato plants against high soil temperatures.

\section{CELL BIOLOGY}

\section{Barbed wires}

Cell 128, 901-913 (2007)

Inside a cell, networks of filaments made from a protein called actin push and pull on membranes to generate shape and movement. Jack Taunton of the University of California, San Francisco, and his colleagues found that a protein required for creating new filaments is also involved in attaching them to membranes.

This protein, known as N-WASP, captures the fast-growing, 'barbed' end of actin filaments and tacks them onto membranes. The authors describe how mutations in two parts of the protein called WH2 domains stop this from happening and cause actin filaments to separate from membranes in vitro.

The team also found that intact versions of these domains were needed for cancer cells to form specialized protrusive structures called podosomes. Taunton says these podosomes may be necessary for cancers to spread around the body.

\section{OCEANOGRAPHY}

\section{Atlantic abatement}

Science 317, 935-938 (2007)

Fears that global warming might be shutting down the circulation of the North Atlantic were allayed by the initial results from an array of instruments that transects the Atlantic between the Canary Islands and Florida.

In the Atlantic deep, cold water flows south while warm, near-surface water flows north, and this 'overturning circulation' is of profound climatic importance. Stuart Cunningham of Britain's National

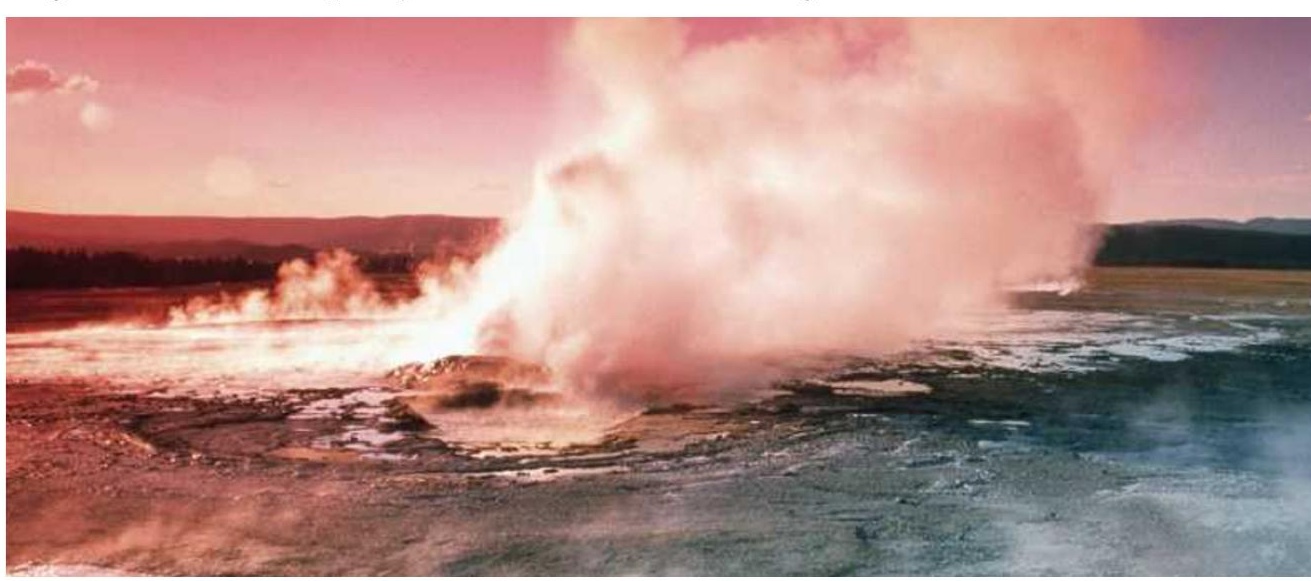


Oceanography Centre in Southampton, Jochem Marotzke of the Max Planck Institute for Meteorology in Hamburg, Germany, and their colleagues accounted for an earlier report of a $30 \%$ decrease in the rate of this overturning, which was published in Nature in 2005 (H. L. Bryden et al. Nature 438, 655-657; 2007). Having studied variations in circulation over time, the researchers write that the earlier conclusion was based on a measurement taken during a natural lull, a possibility that was acknowledged in the original study.

\section{ASTRONOMY}

\section{Sources in the sky}

Science 318, 938-943 (2007)

A collaboration involving more than 440 researchers associated with the Pierre Auger Observatory in Mendoza, Argentina, has traced ultra-high-energy cosmic rays to their apparent source for the first time.

Unlike lower-energy cosmic rays, these ultra-high-energy particles are only marginally deflected by magnetic fields they encounter on the way from their sources to Earth. Using 1,600 ground-based instruments spread across 3,000 square kilometres of Argentina's Pampa Amarilla plain, the Auger team was able to gather enough information on the extremely rare rays to say where in the sky they were coming from. The sources coincide with previously mapped active galactic nuclei (see illustration below), thought to be powered by supermassive black holes.

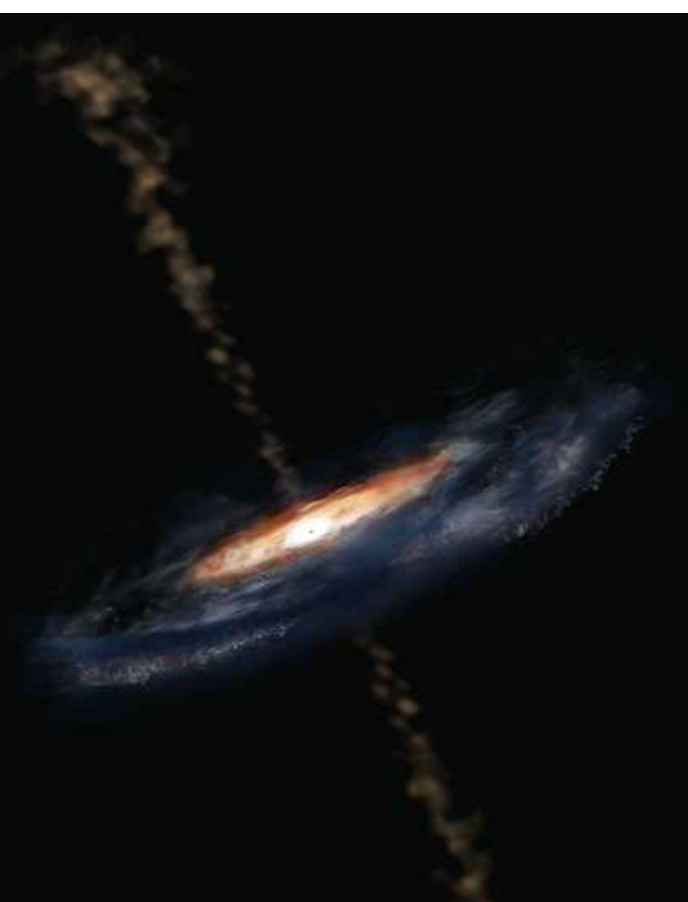

\section{MATHEMATICS}

\section{Mind over Möbius}

Nature Mater. doi:10.1038/nmat1929 (2007) Gert van der Heijden and Eugene Starostin of University College London calculated the 'relaxed' shape of an elastic Möbius strip.

The Möbius strip is the one-sided surface with no ends. The final shape and the potential energy stored in its bends (pictured right) are governed by equations that can be hundreds of pages long.

Poking around Google led the duo to a theory that could be used to drastically simplify the problem by changing the coordinate system in which the calculations are made. The reformulated equations can be used to study a variety of problems, such as the crumpling of paper and the deformation of nanoribbons or twisted biofilaments.

\section{PHYSICS}

\section{Gravity's boost}

Phys. Rev. Lett. 98, 021101 (2007)

Dan Kapner and his colleagues at the University of Washington in Seattle measured the force of gravity between two masses only 55 micrometres apart, roughly the width of a human hair. The force rose as the distances between the masses shrank, in accordance with theory.

In quantum field theory, forces are carried by particles. If the force had fallen away faster than theory had predicted, this could have indicated that the particles associated with gravity are relatively large, and that, in turn, might have explained why cosmologists have found much less cosmological 'dark energy' than quantum theory predicts. These authors' findings eliminate that possibility.

\section{CHEMISTRY}

\section{A simple solution}

Science 317, 1189-1192 (2007)

Our understanding of how molecules will react is usually gleaned from experiments carried out with organic solvents available in standard chemistry labs. For natural products that come from water-living organisms, it might help to think about things differently.
Timothy Jamison and Ivan Vilotijevic at the Massachusetts Institute of Technology in Cambridge have synthesized the core piece of a famously hard-to-make marine molecule by working in neutral water. The molecule, which causes the toxicity associated with 'red tide' algal blooms, has a ladder-like arrangement of rings. It was thought that this structure might assemble through a cascade of reactions, but not until Jamison and Vilotijevic tried it in water did the molecule zip together with ease.

\section{VISION}

\section{A scaffold in} new light

Cell 131, 80-92 (2007)

The fruitfly protein INAD had long been considered to be a scaffolding protein, organizing important visual signalling proteins that attach to it. But recent research suggests that INAD directly regulates visual perception. Rama Ranganathan, of the University of Texas Southwestern Medical Center in Dallas, and his colleagues show that, in response to light, one of five structural 'PDZ' domains of INAD transiently switches from a reduced to an oxidized state, distorting INAD's ability to bind to other molecules. This seems crucial to visually mediated reflex behaviours and for terminating visual responses.

Many scaffolding proteins contain PDZ domains, which could undergo similar conformational changes to that of INAD. Thus, rather than support components, these might serve as control centres for other signalling molecules.

\section{CANCER BIOLOGY \\ Shock strategy}

\section{Cell 130, 1005-1018 (2007)}

A protein called heat-shock factor 1 (HSF1) that helps cells to handle stress seems to have a sinister alter ego. The latest findings suggest that the protein aids tumour growth.

Susan Lindquist of the Whitehead Institute for Biomedical Research in Cambridge, Massachusetts, and her colleagues studied mice that lack the $H s f 1$ gene. These mice developed fewer tumours when they had cancer-linked mutations or when they were exposed to carcinogens than did normal mice. HSF1 also aided human tumour-cell growth in culture.

The results have mixed therapeutic 


\section{Pikes and planning}

Proc. Natl Acad. Sci. USA 104, 15799-15804 (2007)

Fisheries management uses population models that assume fishing does not cause rapid evolutionary change. Nils Stenseth of the University of Oslo and his colleagues found evidence against this premise.

The team analysed data on half a century of pike (Esox lucius, pictured) catches from Lake Windermere in England's Lake District. The records included each pike's age, which can be counted from rings that form in bones around the gills.

Predators normally prefer the smallest fish, whereas humans prize the largest. So fishermen apply an opposing selective pressure from the rest of nature. During periods when fishing was heavy, it became the dominant pressure, and pike tended to be smaller and to devote less energy to reproduction. The findings suggest that fisheries managers should include the evolutionary effects of fishing in their estimates.

implications. They suggest that HSF1inhibiting drugs could combat cancer. But HSF1-stimulating therapies are being explored as treatments for disorders including Parkinson's disease. Researchers will need to explore how each approach affects the problem targeted by the other.

\section{CHEMISTRY}

\section{Getting the right version}

Science 317, 496-499 (2007)

Mirror-image versions of some molecules called chiral - are not structurally identical, and ensuring that only one version is produced in a chemical reaction is often a problem. One way is to use a metal catalyst attached to a ligand molecule that is also chiral.

Ligands are normally attached to metal catalysts by strong covalent bonds, but Dean Toste and his colleagues at the University of California, Berkeley, have successfully used

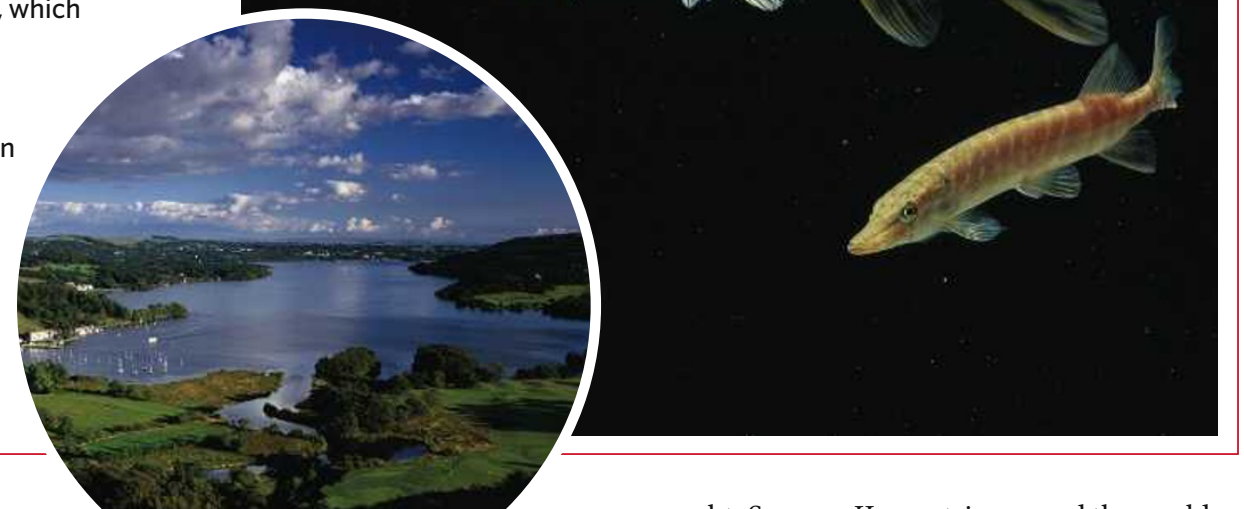

yacht, Sorcerer II, on a trip around the world (route pictured below left) contained gene fragments predicted to represent more than six million proteins. In March, when the work was published, that was enough to almost double the number of proteins listed in online databases. The data set included new members of nearly all known protein families from bacteria and archaea, and a large number of viral genes, suggesting viral diversity has to date been undersampled.

\section{MOLECULAR NEUROBIOLOGY \\ Active resilience}

\section{Sorcerer's survey}

PLoS Biol. 5, e16 (2007)

Shibu Yooseph of the J. Craig Venter Institute in Rockville, Maryland, and his colleagues have revealed more of the oceans' genetic diversity than has ever previously been seen.

Sea-water samples collected by Venter's

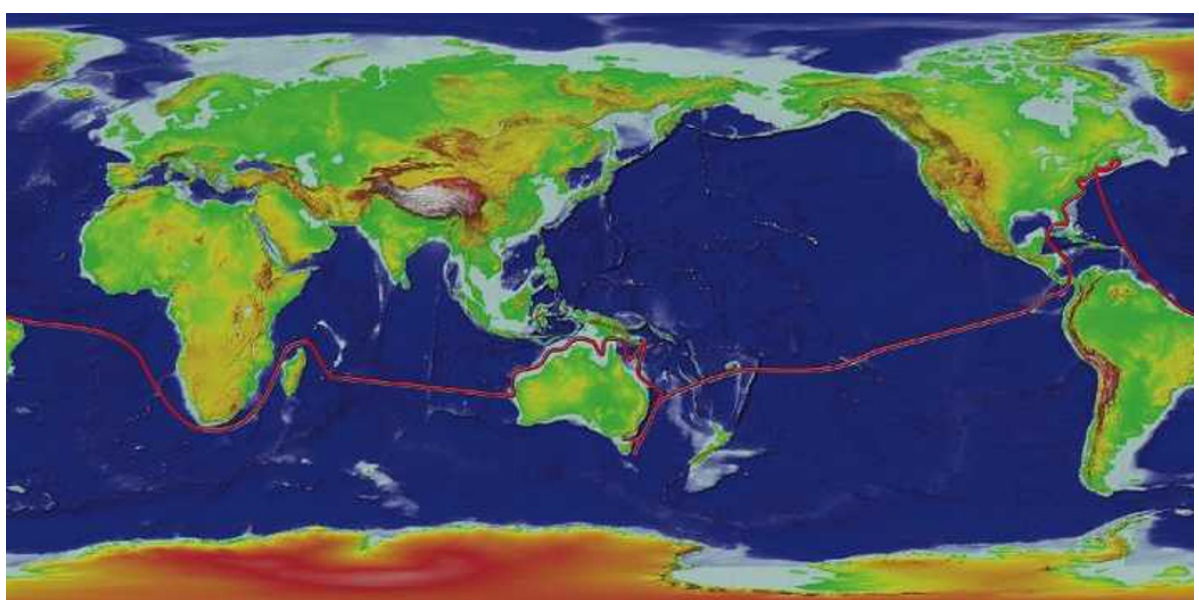

Cell 131, 391-404 (2007)

A molecule known as BDNF may provide a pivotal distinction between people who succumb to conditions such as depression or post-traumatic stress disorder and the majority who do not.

Eric Nestler of the University of Texas Southwestern Medical Center in Dallas and his team had previously identified mice that avoided social contact with cage-mates after repeated 'social defeat' - brought about by forced encounters with more aggressive mice.

The researchers found that resilience to stress in this social-defeat model is an active process in which stress-induced firing in part of the brain's neuronal circuitry associated with reward and drug addiction is suppressed. This lowers levels of BDNF (brain-derived neurotrophic factor), and mice with a natural variation in their BDNF gene were resilient to stress. The researchers also found high levels of BDNF in a region associated with reward in post-mortem brains of people with a history of depression. 